\title{
Thermodynamic Potentials and Natural Variables
}

\author{
M. Amaku ${ }^{1,2}$, F. A. B. Coutinho ${ }^{* 1}$, L. N. Oliveira包 \\ ${ }^{1}$ Universidade de São Paulo, Faculdade de Medicina, São Paulo, SP, Brasil \\ ${ }^{2}$ Universidade de São Paulo, Faculdade de Medicina Veterinária e Zootecnia, São Paulo, SP, Brasil \\ ${ }^{3}$ Universidade de São Paulo, Instituto de Física de São Carlos, São Carlos, SP, Brasil
}

Received on May 30, 2019. Revised on September 13, 2018. Accepted on October 4, 2019.

\begin{abstract}
Most books on Thermodynamics explain what thermodynamic potentials are and how conveniently they describe the properties of physical systems. Certain books add that, to be useful, the thermodynamic potentials must be expressed in their "natural variables". Here we show that, given a set of physical variables, an appropriate thermodynamic potential can always be defined, which contains all the thermodynamic information about the system. We adopt various perspectives to discuss this point, which to the best of our knowledge has not been clearly presented in the literature.
\end{abstract}

Keywords: Thermodynamic Potentials, natural variables, Legendre transforms.

\section{Introduction}

Basic concepts are most easily understood when we discuss simple systems. Consider an ideal gas in a cylinder. The cylinder is closed, its walls are conducting, and a fluid at fixed temperature $T$ surrounds it. The cylinder contains a fixed number $N$ of particles. Its volume $V$ can nonetheless be varied, by means of a plunger. We push the plunger and do work on the gas. Heat flows out to maintain the gas at the temperature $T$. If, instead, we slowly pull out the plunger, heats flows inwards.

In experiments, the pressure $P$ applied to the plunger can be controlled, and the fluid temperature $T$ can also be controlled. The variables $P, T$ and $V$ are not independent. It has been known for centuries that this system approximately obeys an equation of state, which binds the three variables:

$$
P V=N k_{B} T
$$

where $k_{B}$ is Boltzmann's constant.

One might think that Eq. (1) is all we need to describe the ideal gas. The equation of state, nonetheless, tells us nothing about other important properties of the system. The Internal Energy $U$ rises or drops as the temperature changes. We might also be interested in the entropy $S$, and so forth.

The ideal gas is a mathematical concept: a collection of particles moving independently from each other and bouncing elastically off the cylinder walls. The simple dynamics makes $U$ directly proportional to the temperature and number of particles. The proportionality to $N$, a variable that - except as explicitly indicated - we will keep fixed, is an inherent property of the internal energy. The

*Endereço de correspondência: coutinho@dim.fm.usp.br same statement cannot be applied to the temperature. In real fluids, even in simple ones, the proportionality to $T$ is washed out, and the Internal Energy is more conveniently expressed as a function of the entropy and volume: $U=U(S, V)$.

Experimentalists frown at this dependence, for entropies are more difficult to measure than temperatures. Moreover, they may have to let the plunger move back and forth and adjust the cylinder pressure to that of the environment. They would therefore prefer the variables $P$ and $T$, instead of $S$ and $V$.

At this point, it would seem natural to say "Very well, let us make the experimentalist happy. Determine the entropy and volume as functions of $T$ and $P$. Since $U$ is known as a function of $S$ and $V$, all we have to do, then, is to substitute $S(T, P)$ and $V(T, P)$ for $S$ and $V$. The result will be $U[S(T, P), V(T, P)]$, which expresses $U$ as a function of $T$ and $P$."

But then we would be at odds with good textbooks, which prefer to define another extensive quantity, on equal footing with the internal energy: the Gibbs Free Energy $G(T, P)$, a function of the experimentalist's favorite variables. The Internal Energy and the Gibbs Free Energy are thermodynamic potentials. As we shall see, a simple expression relates $G(T, P)$ to $U(S, V)$. The Gibbs Free Energy $G$ must be expressed as a function of $T$ and $P$, just like the Free Energy $U$ must be expressed as a function of $S$ and $V$. The temperature $T$ and the pressure $P$ are called the natural variables of $G$. The natural variables of $U$ are $S$ and $V$.

Once again, our inner voice interrupts the train of thoughts to argue in favor of the experimentalist: "This is all very well, but $G$ is obtained from $U$. Therefore, in order to compute $G(T, P)$, we will first have to express the entropy and volume as functions of temperature and 
pressure to compute $U[S(T, P), V(T, P)]$. Our friend, the experimentalist, wants the Internal Energy as a function of $T$ and $P$. So give her $U[S(T, P), V(T, P)]$. Why waste time computing $G(T, P)$ ? Who needs the Gibbs Free Energy?"

Textbooks on Thermodynamics define the thermodynamic potentials and associate them with their natural variables. A few texts offer indirect answers to the two questions we have posed. To explain why $S$ and $P$ are the natural variables of the Enthalpy, Schroeder shows that changes in $H$ have simple physical interpretation when the pressure is held fixed 1]. To explain why $T$ and $P$ are the natural variables of the Gibbs Free Energy, Callen shows that, for a system in contact with a pressure and a temperature reservoirs, the equilibrium value of any of its internal parameters will minimize $G$ at the reservoir pressure and temperature 2. Callen also explains that information is lost when a thermodynamic potential is expressed not as a function of its natural variables, but as a function of its derivative with respect to those variables 2]. To prop up the argument, the author presents a geometrical depiction of this problem and of its solution by means of Lagrange Transformations. This explanation pleases experienced teachers. To the same extent, it disappoints the students, who often regard the illustration as an abstract solution to an abstruse conundrum.

The purpose of this paper is to answer the two questions directly, in a physical setting, that is, to explain why it is better to work with $G(T, P)$ than with $U[S(T, P), V(T, P)]$. We will show that $U[T, P] \equiv U[S(T, P), V(T, P)]$ conveys less information than $U(S, V)$, or $G(T, P)$. In order to preserve information, we have to express each thermodynamic potential as a function of its natural variables. Conversely, the variables of interest uniquely determine the thermodynamic potential. In our example, the experimentalist would like to work with $T$ and $P$. She should then ask for $G(T, P)$, not for $U(T, P)$.

\section{Four thermodynamic potentials}

As explained in Sec. 11 four physical variables are associated with the gas in the cylinder: $T, P, S$, and $V$. When these variables are increased or reduced, the thermodynamic potentials $U$ and $G$ change. The changes $d U$ in the Internal Energy are especially simple to describe, since the First Law of Thermodynamics states that any $d U$ is a linear combination of the changes in its natural variables 3],

$$
d U=T d S-P d V
$$

with linear coefficients $T$ and $P$.

Given $U(S, V)$, Eq. (2) yields the temperature

$$
T=\left.\frac{\partial U(S, V)}{\partial S} \equiv \frac{\partial U}{\partial S}\right|_{V}
$$

and the pressure

$$
P=-\frac{\partial U(S, V)}{\partial V} \equiv-\left.\frac{\partial U}{\partial V}\right|_{S} .
$$

Here we have adopted the traditional notation [2, 4], which explicitly indicates the fixed quantity defining the partial derivative. Since we work with fixed number of particles, to simplify the notation we have avoided adding a second, redundant subscript $N$ to the partial derivatives.

In view of Eq. (3), $T$ and $S$ are said to be conjugate variables. Likewise, in view of Eq. (4), $P$ and $V$ are conjugates. In a conjugate pair, one of the variables ( $T$ or $P)$ is intensive, independent of the number of particles, while the other $(S$ or $V$ ) grows in proportion to the number of particles and is, hence, extensive.

The dependence on extensive variables makes $U$ somewhat special among the thermodynamic potentials. That the Internal Energy is a (single-valued) convex function of all its natural variables, $S$ and $V$, was first pointed out by Gibbs. Given the convexity and that $S$ and $V$ are extensive, it is a simple matter to derive the Second Law of Thermodynamics, as recalled by Wightman, in a remarkably clear, succinct account of Gibbs's reasoning [5]. The other thermodynamic potentials cannot be directly related to the Second Law because they depend on one or more intensive variables; while they are convex functions of the extensive variables, they are concave function of the intensive ones 2 .

Nonetheless, practical considerations often focus one's interest on intensive variables. One must then turn to thermodynamic potentials other than $U(S, V)$. We have already discussed one of them, the Gibbs Free Energy $G(T, P)$. Since we have four variables $(S, V, T$, and $P)$, there must be more than two thermodynamic potentials, and can ask for the number of alternatives.

As illustrated by Eq. (2), the differential of a thermodynamic potential is a linear combination comprising the differentials of its natural variables; the linear coefficients are the conjugates to the natural variables. Out of the two conjugate pairs $(S, T)$ and $(V, P)$, we can construct four pairs of natural variables: (i) $S$ and $V$, (ii) $S$ and $P$, (iii) $T$ and $V$, and (iv) $T$ and $P$. We have, therefore, four thermodynamic potentials. Two of them were introduced in Sec. 1. The other two are the Helmoltz free energy $F(T, V)$ and the enthalpy $H(S, P)$.

\subsection{Expressions for the four thermodynamical potentials}

We now describe the schematic procedure determining each of the thermodynamic potentials in Table 1 . The procedure is schematic because it does not explain how the resulting quantities can be expressed as functions of the pertinent natural variables, a task discussed in Sec. 2.2

Consider the Helmholtz Free Energy, the thermodynamic potential whose natural variables are $T$ and $V$. Our 
analysis starts with the First Law of Thermodynamics, Eq. (2), which expresses changes in the Internal Energy as a linear combination of the changes in $T$ and $V$. We want to substitute the variable $T$ for $S$. To this end, we subtract $d(T S)$ from the right-hand side of Eq. (2), to show that

$$
d F=d U-d(T S)
$$

from which we obtain an expression for the Helmholtz Free Energy:

$$
F=U-T S
$$

Moreover, from Eqs. (2) and (5), it follows that

$$
d F=-S d T-P d V
$$

which expresses changes in the Free Energy as a linear combination of the changes in its natural variables, $T$ and $V$.

Next, we want to calculate the Enthalpy, $H=H(S, P)$. We want, therefore, to substitute a term proportional to $d P$ for the term proportional to $d V$ on the right-hand side of Eq. 2). We then write

$$
d H=d U+d(P V)
$$

which shows that

$$
H=U+P V
$$

Equalities such as Eq. (8) have simple physical interpretations. An Enthalpy change $\Delta H$ at constant pressure is the sum of the associated internal-energy change $\Delta U$ with the work $P \Delta V$ that must be done on the surroundings to vacate the volume $\Delta V$. One can likewise interpret all the other thermodynamics potentials, as explained by Schroeder 1].

From the practical viewpoint, it is more important to express the changes in the Entropy as a linear combination of the changes in its natural variables, $S$ and $P$. With this goal in mind, we substitute the right-hand side of Eq. (2) for $d U$ on the right-hand side of Eq. (8), which yields the expression

$$
d H=T d S+V d P .
$$

Experimentalists find Eq. 10 very convenient. Working at fixed pressure, they only have to measure the heat $d Q=T d S$ that is exchanged with the environment to determine the change in Enthalpy.

Finally, to determine the Gibbs Free Energy $G(T, P)$, we add $d(P V)$ to and subtract $d(T S)$ from the right-hand side of Eq. (2):

$$
d G=d U-d(T S)+d(P V)
$$

which shows that

$$
G=U-T S+P V
$$

Substitution of the right-hand side of Eq. 2 for $d U$ on the right-hand side of Eq. (11) then shows that

$$
d G=-S d T+V d P .
$$

As we have already pointed out, these manipulations are schematic. To make the equalities for $F, H$, and $G$ practical, we must express the right-hand sides of Eqs. (6), $(9)$, and $(12)$ as functions of the natural variables $(T, V)$, $(S, P)$, and $(T, P)$, respectively. Section 2.2 explains how this can be done.

\subsection{Expression of the four thermodynamical potentials as functions of their natural variables.}

Consider, as an example, the expression for the Helmholtz Free Energy derived in Sec. 2.1. Eq. (6). The first term on the right-hand side, $U$, is known as a function of the entropy and volume. Since we are interested in the Free Energy, we want to express $U$, and also the entropy, which is a factor in the last term on the right-hand side, as functions of $T$ and $V$. The following sequence solves our problem:

Step 1 From $T=\left(\frac{\partial U}{\partial S}\right)_{V}$, find $T=T(S, V)$;

Step 2 Invert the expression obtained in Step 1 to find $S=S(T, V)$

Step 3 Substitute $S(T, V)$ for $S$ on the right-hand side of Eq. (6) to find $F(T, V)$.

As we shall see in Sec. 4.2, no information is lost in this procedure. Equation (6), which defines the Helmholtz Free Energy, is a Legendre Transformation, a procedure designed to ensure that the resulting potential $[F(T, V)]$ contain the same information as the original one $[U(S, V)]$.

Analogous algebra reduces Eq. (9) to an expression for $H(S, P)$, and Eq. 12 to an expression for $G(T, P)$. For a more detailed presentation, see Zia et al. 6 .

The left-hand column of Table 1 collects the central results inSec. 2.1. For each thermodynamic potential $U$, $F, H$, and $G$, the right-hand column presents the expressions for the two conjugate variables directly obtained from Eqs. (2), (7), 10), and (13), respectively.

\section{Multiplicity of the thermodynamic potentials}

Equation (2) covers systems with only two degrees of freedom. It suits the gas in our cylinder well, which can be described by two independent intensive variables: the temperature $T$ and pressure $P$. This, however, is not the most general situation.

Consider, for instance, instead of the gas in the cylinder, a stretchable wire. Its volume can be varied and hence defines an additional degree of freedom. Let $X=L-L_{0}$ 


\begin{tabular}{ccc}
\hline Potential & Natural independent variables & Conjugate dependent variables \\
\hline INTERNAL ENERGY & $S, V$ & $\left.P=-\left(\frac{\partial U}{\partial S}\right)_{V}\right)_{S}$ \\
\hline HELMHOLTZ FREE ENERGY & $T, V$ & $S=-\left(\frac{\partial F}{\partial T}\right)_{V}$ \\
$F=U-T S$ & $P=-\left(\frac{\partial F}{\partial V}\right)_{T}$ \\
\hline ENTHALPY & $T, P$ & $T=\left(\frac{\partial H}{\partial S}\right)_{P}$ \\
$H=U+P V$ & & $V=\left(\frac{\partial H}{\partial P}\right)_{S}$ \\
\hline$G=H-T S$ & $S, P$ & $V=-\left(\frac{\partial G}{\partial T}\right)_{P}$ \\
\hline
\end{tabular}

Table 1: The four thermodynamic potentials in Sec. 2 and the two conjugate physical variables determined from each.

be the length of the wire, measured from its equilibrium length $L_{0}$, and $V$ be its volume. Let $f$ be the force stretching the wire, and let $T$ and $P$, as before, be the temperature and pressure. Equation (2) must then be extended to the form

$$
d U=T d S-P d V+f d X
$$

In correspondence with the three degrees of freedom, we now have three intensive variables, $T, P$ and $f$, and Eq. (14) neatly pairs each one with an extensive variable. In this case, there are three conjugate pairs: $(S, T)$, $(V, P)$, and $(X, f)$. Out of them we can construct eight thermodynamic potentials, which are functions of (i) $S, V$ and $X$, (ii) $S, V$ and $f$, (iii) $S, P$ and $X$, (iv) $S, P$ and $f$, (v) $T, V$ and $X$, (vi) $T, V$ and $f$, (vii) $T, P$ and $X$, and (viii) $T, P$, and $f$.

More generally, the number $n$ of intensive variables, or of degrees of freedom, determines the number of thermodynamic potentials. The number of potentials is $2^{n}$. Although this can be readily infered from the above reasonings, for $n=2$ and $n=3$, we refer the more skeptical reader to Adkins [7, page 111], for a formal proof.

An important example in the $n=3$ category is the extension of the thermodynamic potentials in Table 1 to systems with variable number of particles. The intensive variable conjugate to $N$ is the chemical potential $\mu$. Just as it is convenient to work with the temperature instead of the entropy, it may be convenient to consider $\mu, T$, and $V$ as variables, instead of $N, S$, and $V$. Algebraic manipulations analogous to the ones in Sec. 2.1 then define the Grand Potential

$$
\Omega(\mu, T, V)=F(N, T, V)-\mu N,
$$

one of the thermodynamic potentials associated with the conjugate pairs $(S, T),(V, P)$, and $(N, \mu)$.

On the basis of our discussion, above, we might expect to define altogether eight thermodynamic potentials from the four conjugate pairs. As Sec. 6 will explain, however, one of the functions vanishes identically. The number of thermodynamic potentials is, therefore, seven.

\section{Thermodynamic potentials and natural variables.}

We now have to come to grips with the question in Sec. 1 which bothers every serious student of Thermodynamics. Given a thermodynamic potential, why must it be expressed as a function of its natural variables? Two answers can be offered.

\subsection{Natural variables give easy access to all physical properties.}

The first answer can be found, for example, in Baierlein 3 page 236], which shows that the natural variables are special because they are the only ones giving immediate access to the other physical variables. Table 1 shows how $T$ and $P$ can be obtained from $U(S, V)$, how $S$ and $P$ can be obtained from $F(T, V)$, and so forth. Would it be possible to likewise extract $S$ and $V$ from $U(T, P)$ ? The answer is negative. 
The difficulty is not to express $U$ as a function of $T$ and $V$. Suppose that we know $U(S, V)$. Then, from Eq. (3), we can determine the temperature as a function of the entropy and volume: $T=T(S, V)$. Likewise, from Eq. (4) we can determine $P=P(S, V)$. Inversion of these two equalities yields $S=S(T, P)$ and $V=(T, P)$. Substitution in the arguments of our expression for $U(S, V)$ finally yields $U[S(T, P), V(T, P)] \equiv U(T, P)$. That is as far as we can go, however, because there is no simple way to compute the other physical variables, $S$ and $V$, from $U(T, P)$. By comparison, $U(S, V)$ is much more convenient.

It is, of course, possible to choose $T$ and $P$ as natural variables. For that, however, one must define the appropriate thermodynamic potential, the Gibbs Free Energy $G(T, P)$. From $G(T, P)$, the partial derivatives in Table 1 yield the other physical variables.

One might follow an altogether distinct program and measure, rather than compute, the internal energy $U$ as a function of variables other than $S$ and $V$. Even then, however, the other variables cannot be obtained from the partial derivatives of the resulting $U$, simply because the derivative of a function $f$ does not uniquely determine $f$, as pointed out by Callen [2]. Nor can we obtain the other thermodynamic potentials from $U(T, P)$, for instance, as Section 4.2 will explain.

\subsection{From a thermodynamic potential expressed as function of its natural variables, all the other potentials can be recovered.}

We now turn to another answer to the question raised in Sec. 1 If a potential is measured as a function of variables other than its natural variables, one might hope that adequate mathematical treatment would allow one to express the same thermodynamic potential as a function of its natural variables. If so, one could then derive the other variables from the latter expression. Unfortunately, that is not possible, as we now shall show.

Let us assume, for example, that $U$ has been measured as a function of $T$ and $P: U=Y(T, P)$. We want $U(S, V)$. To this end, we take advantage of the following relations, from Table 1 :

$$
T=\left(\frac{\partial U}{\partial S}\right)_{V}
$$

and

$$
-P=\left(\frac{\partial U}{\partial V}\right)_{S},
$$

to write the partial differential equation

$$
U=U(S, V)=Y\left(\left(\frac{\partial U}{\partial S}\right)_{V},\left(\frac{\partial U}{\partial V}\right)_{S}\right) .
$$

To solve Eq. (18), we would need boundary conditions [8]. By itself, the function $Y(T, P)$ cannot pro- vide such conditions. We would therefore need additional information to determine $U(S, V)$. This is in striking contrast with the procedure that expresses $T$ and $P$ as functions of $S$ and $V$ and hence, provided only that the resulting expressions can be inverted so that $S$ and $V$ be found as functions of $T$ and $P$, determines $Y(T, P)=U[S(T, P), V(T, P)]$. The road leading from $U(S, V)$ to $Y(T, P)$ is a one-way street. This unexpected asymmetry calls for an explanation, to which we now turn, in a simpler context.

Suppose we are given a function

$$
F=F(x)
$$

and want to generate a new function $G$, of the variable

$$
y=\frac{d F(x)}{d x}=F^{\prime}(x)
$$

in such a way that from $G(y)$ we can recover $F(x)$.

We will assume, additionally, that Eq. 20 can be inverted:

$$
x=g(y)
$$

Inversion is, of course, a simple matter if the relation expressed by Eq. 19 is the result of an extensive number of measurements.

One might think that our problem has a simple solution [2]: all we have to do is to substitute $g(y)$ for $x$ on the right-hand side of Eq. $\sqrt{19}$ to generate an ordinary differential equation for $F(x)$.

$$
F=F(x)=F(g(y))=G\left(\frac{d F(x)}{d x}\right) .
$$

Unfortunately, Eq. 22 is insufficient to determine $F(x)$. There are infinitely many solutions, which differ from each other by a constant. Since that constant is unknown, we cannot find $F(x)$. Information was lost when we switched variables, from $x$ to $y$.

We have likewise lost information in our derivation of Eq. (18). The solutions to Eq. (18) differ from each other by arbitrary functions. We would therefore have to know the pertinent function to determine the internal energy $U(S, V)$ from $U(T, P)$.

The Legendre transformation offers an elegant solution to this problem. To describe the procedure, we try again to generate a function $G(y)$, with $y$ given by Eq. (20), such that $F(x)$ can be directly retrieved from it.

The Legendre transform of the function $F(x)$ is defined by the equality

$$
L\{F(x)\}=x \frac{d F(x)}{d x}-F(x) .
$$

Reference to Eqs. (20) and (21) yields the alternative expression

$$
L\{F(x)\}=g(y) y-F(g(y))
$$


which defines the transformed function

$$
G(y) \equiv g(y) y-F(g(y)) \text {. }
$$

It is now a simple matter to show that $G(y)$ retains the information in $F(x)$. We only have to compute the Legendre transform of $G(y)$, in analogy with Eq. (23):

$$
L\{G(y)\}=y \frac{d G(y)}{d y}-G(y) .
$$

The derivative in the first term on the right-hand side of Eq. 26) can be extracted from Eq. 25):

$$
\frac{d G(y)}{d y}=\frac{d g(y)}{d y} y+g(y)-\frac{d F(g)}{d g} \frac{d g(y)}{d y} .
$$

Equation 20 now reminds us that the derivative $d F(g) / d g$ in the second term on the right-hand side of Eq. 27) is our variable $y$. With this, the latter equality reduces to the form

$$
\frac{d G(y)}{d y}=g(y)
$$

and since $g(y) \equiv x$, we conclude that Eq. (26) is equivalent to the expression

$$
L\{G(y)\}=y x-G(y) .
$$

Substitution of the right-hand side of Eq. 25 for $G(y)$ on the right-hand side of Eq. 29 then shows that

$$
L\{G(y)\}=F(g(y)) .
$$

We can see that the Legendre transform of $G(y)$ brings us back to $F(x)$. The Legendre transform is involutive: the transform of the transform is the original function. More importantly for our purposes, the function $G(y)$ contains exactly the same information as $F(x)$.

The First Law of Thermodynamics, given by equalities such as Eq. (2) or Eq. (14), depending on the number of degrees of freedom, identifies the natural variables for the internal energy $U$. For example, the natural variables for the wire associated with Eq. (14) are $S, V$, and $X$. The Legendre transforms of $U$ are the seven thermodynamic potentials whose natural variables are the partial derivatives of $U$ with respect to $S, V$, or $X$. Another example, derived from Eq. (2), is found in Table 1 the last three rows in its first column displays the three thermodynamic potentials defined by the Legendre transforms of $U(S, V)$.

\subsection{Adoption of variables other than the natural ones calls for additional information}

Sections 4.1 and 4.2 have argued that the expression of a thermodynamic potential as a function of variables other than its natural variables contains less information than the expression of the same potential as a function of its natural variables. Here, we will discuss two examples. In the first example, the Third Law of Thermodynamics offers the missing information. In the second, the Third Law is insufficient to fill the gap.

\subsubsection{Internal energy as a function of $T$ and $V$}

The first illustration considers an experiment in which the internal energy has been measured as a function of volume and temperature 9. It results that $U=v(T, V)$. Inspection to Table 1 shows that $V$ is a natural variable of $U$, while $T$ is not. We would therefore like to obtain $U(S, V)$ from the function $v(T, V)$. This, as we shall see, is only feasible because the Third Law of Thermodynamics provides the missing information.

The procedure determining $U(S, V)$ comprises four steps:

1. Find the potential with natural variables $T$ and $V$. Table 1 indicates that the desired potential is the free energy $F(T, V)$. We will try to find $F(T, V)$ and then Legendre transform it to obtain $U(S, V)$.

2. Relate the function identified in step 1 to the internal energy, care being taken to eliminate any variables other than $T$ and $V$ from the relation. The first cell in the second row of Table 1 shows that

$$
U=F+T S .
$$

With help of the third cell in the same row, we then see that

$$
U=F-T\left(\frac{\partial F}{\partial T}\right)_{V} .
$$

3. Convert the result into an explicit expression for the potential in step 1 as a function of the variables $T$ and $V$. We divide both sides of Eq. (32) by $T^{2}$ to show that

$$
\frac{\partial(F / T)}{\partial T}=-\frac{U}{T^{2}}
$$

Integration of both sides of Eq. (33) now yields the sought expression:

$$
F(T, V)=-T \int_{0}^{T} \frac{v\left(V, T^{\prime}\right)}{T^{\prime 2}} d T^{\prime}+\phi(V) T .
$$

As expected, this result calls for additional information, to determine the unknown function $\phi(V)$.

4. Use additional information to determine the thermodynamic potential. The Third Law of Thermodynamics states that the entropy vanishes at $T=0$, for any volume. To take advantage of this information, we differentiate both sides of Eq. (34) with respect to $T$. Since $S=-(\partial F / \partial T)_{V}$, we can then see that

$$
S(T, V)=\int_{0}^{T} \frac{v\left(V, T^{\prime}\right)}{T^{\prime 2}} d T^{\prime}+\frac{v(V, T)}{T}-\phi(V) .
$$

From the Third Law of Thermodynamics, we know that the left-hand side of Eq. 35 vanishes as $T \rightarrow 0$. 
It follows that

$$
\phi(V)=\lim _{T \rightarrow 0}\left(\frac{v(V, T)}{T}\right),
$$

which brings Eq. (34) to the closed form

$F(T, V)=-T \int_{0}^{T} \frac{v\left(V, T^{\prime}\right)}{T^{\prime 2}} d T^{\prime}+T \lim _{T \rightarrow 0}\left(\frac{v(V, T)}{T}\right)$.

We can then Legendre transform $F(T, V)$ to determine $U(S, V)$.

Ideal gas The internal energy of an ideal gas is independent of volume and proportional to the temperature. The internal energy of a monoatomic gas, for instance, is given by the equation

$$
U=v(T, V)=\frac{3}{2} N k_{B} T .
$$

With this function, the integral in the first term on the right-hand side of Eq. (37) diverges at the lowerlimit. Equation (37) is, therefore, inapplicable. One may find that hardly surprising, given that the ideal gas is a mathematical model that was developed well before the Third Law of Thermodynamics.

The more inquisitive reader will nonetheless be disappointed in the inadequacy of Eq. (37). She will be pleased to know that an expression derived when Quantum Mechanics was still under construction eliminates the divergence. The entropy of the ideal gas was determined in 1912, by Otto Sackur and Hugo Tetrode. Enlightening, comprehensive accounts of their work have been presented by Grimus [10]. The Sackur-Tetrode equation reads

$$
\begin{aligned}
S(U, V, N)=k_{B} N & \left(\frac{3}{2} \log \frac{U}{N}+\log \frac{V}{N}\right. \\
+ & \left.\frac{3}{2} \log \frac{4 \pi m}{3 h^{2}}+\frac{5}{2}\right),
\end{aligned}
$$

where $h$ and $m$ are Planck's constant and the particle mass, respectively.

Equation 39 presents the entropy as a function of the Internal Energy and volume, and hence deserves a brief digression. Inversion yields the equality

$$
U(S, V)=\frac{3 h^{2}}{4 \pi m} e^{\left(\frac{2}{3} \frac{S}{N k_{B}}-\frac{5}{3}\right)}\left(\frac{V}{N}\right)^{-\frac{2}{3}},
$$

which expresses the Internal Energy as a function of its natural variables, $S$ and $V$. The equalities in the last cell in the first row on Table 1 then determine the temperature and pressure.

We can now go back to the problem of computing the ideal-gas Helmholtz Free Energy from Eq. (37). Substitution of the right-hand side of Eq. (38) for $U$ on the right hand side of Eq. $(39)$, yields the following expression for the entropy as a function of temperature and volume

$$
\begin{aligned}
S(T, V, N)=k_{B} N & \left(\frac{3}{2} \log k_{B} T+\log \frac{V}{N}\right. \\
& \left.+\frac{3}{2} \log \frac{2 \pi m}{h^{2}}+\frac{5}{2}\right),
\end{aligned}
$$

which can be rewritten in the form

$$
S(T, V, N)=k_{B} N\left(\log \left(\frac{V}{N \lambda_{T}^{3}}\right)+\frac{5}{2}\right),
$$

where

$$
\lambda_{T} \equiv \frac{h}{\sqrt{2 \pi m k_{B} T}}
$$

is the de Broglie thermal length of the gas particles.

The factor $\lambda_{T}^{3}$ in the denominator of the logarithm on the right-hand side of Eq. 42 can be regarded as the volume of a cube with side equal to the de Broglie length $\lambda_{T}$. The entropy of the ideal gas measures the number of scales separating the per-particle volume $V / N$ from the volume of the de Broglie cube. Consider, for example, a gas of He atoms under standard temperature and pressure conditions. The per-particle-volume is the ratio between the molar volume and Avogadro's number, and the de Broglie length can be computed from Eq. 43). It results that $V / N=4 \times 10^{-26} \mathrm{~m}^{3}$ and $\lambda_{T}^{3}=1.0 \times 10^{-31} \mathrm{~m}^{3}$. The two volumes are therefore split by five orders of magnitude, and the per-particle entropy is substantial.

As the gas is cooled at fixed volume, the de Broglie length grows. At a sufficiently low temperature $T_{0}$, the de Broglie cube becomes larger than $V / N$ by a factor $\exp (5 / 2)$, and the right-hand side of Eq. (42) vanishes. If $\mathrm{He}$ were an ideal gas, its entropy would vanish at $T_{0}^{\mathrm{He}}=1 \times 10^{-2} \mathrm{~K}$.

Back to Eq. (37), we can now see that the lower limit of the integral on the right-hand side should be $T=T_{0}$, not $T=0$. This infrared cutoff ensures convergence of the integral and determines the Free Energy.

\subsubsection{Free energy as a function of temperature and pressure.}

The derivation of Eq. (37) relied on information provided by the Third Law of Thermodynamics. We now show that the Third Law is not always sufficient to provide the information that is missing from the expression of a thermodynamic potential as a function of variables other than the natural ones. In fact, since the Third Law determines a property (the entropy) at a given temperature $(T=0)$ and any volume $V$, the information it provides is only sufficient to calculate the Free Energy, whose natural variables are $T$ and $V$.

Imagine now, for contrast, that the Free Energy be known as a function of pressure and volume, $F=Y(P, V)$, instead of its natural variables $T$ and $V$. It seems advisable to follow the prescription that proved successful in our derivation of Eq. (37). 
In other words, we try to determine the Gibbs Free Energy $G$, whose natural variables are $P$ and $V$. From Table 1 we can see that

$$
Y(T, P)=G(T, P)-P\left(\frac{\partial G}{\partial P}\right)_{T},
$$

both sides of which can be divided by $P^{2}$ to yield the equality

$$
\frac{\partial(G / P)}{\partial P}=-\frac{Y(T, P)}{P^{2}} .
$$

Integration over pressure then yields an explicit expression for the Gibbs Free Energy:

$$
G(T, P)=P \int_{0}^{P} \frac{Y\left(T, P^{\prime}\right)}{P^{\prime 2}} d P^{\prime}+P \bar{\phi}(T),
$$

where $\bar{\phi}$ denotes an unknown function.

To determine the function $\bar{\phi}(T)$ on the right-hand side of Eq. 46, we need additional information. We may try to extract that information from the Third Law of Thermodynamics. For that, we have to turn Eq. 46 into an expression for the entropy. We therefore differentiate both sides with respect to $T$ and recall, from Table 1 . that $S=(\partial G / \partial T)_{P}$. It results that

$$
\begin{array}{r}
S(T, P)=-P \int_{0}^{P} \frac{1}{P^{\prime 2}}\left(\frac{\partial Y\left(T, P^{\prime}\right)}{\partial T}\right)_{P^{\prime}} d P^{\prime} \\
-\left.P \frac{d \bar{\phi}}{d T}\right|_{T} .
\end{array}
$$

At $T=0$, the left-hand side vanishes, and Eq. (47) reduces to an equality that determines the derivative $d \bar{\phi} / d T$ at $T=0$. At low temperatures, substitution of the Taylor expansion for $\bar{\phi}(T)$ on the right-hand side of Eq. (46) therefore yields an approximate expression for $G(T, P)$, within an unknown additive constant. At higher $T$, however, that expression becomes unreliable, and Eq. 46, essentially useless. Only at low temperatures does the Third Law of Thermodynamics determine the Gibbs Free Energy.

\section{Maxwell's relations}

Even when none of the potentials for the system of interest are known, in either analytic or tabular form, we can take advantage of the thermodynamic potentials, because we know their differentials to be exact. The differential of the Internal Energy, for instance, is given by Eq. (2), from which follow the two equalities in the first-row, lastcolumn cell on Table 1 . Since $U$ is an analytic function of its natural variables, partial differentiation of the first equality with respect to $V$ and of the second with respect to $S$ yields the expression

$$
\left(\frac{\partial T}{\partial V}\right)_{S}=-\left(\frac{\partial P}{\partial S}\right)_{V}
$$

Analogous expressions can be derived from the other thermodynamic potentials and, together with Eq. (48), are known as Maxwell's Relations. They show that the physical variables associated with a system cannot be varied independently, because they are constrained by Thermodynamics. Given their conceptual relevance, Maxwell's Relations are extensively discussed in textbooks.

More significant from the practical viewpoint are equalities relating physical properties, such as heat capacities, to other measurable coefficients. We shall discuss two illustrations.

Our first illustration focuses on the Internal-Energy differential. Consider, again, the system described in Section 1, a gas in a cylinder. If its temperature is increased by $d T$, the gas will absorb a differential amount of heat:

$$
d Q=C_{V} d T+\lambda d V
$$

We have chosen the independent variables $T$ and $V$. The coefficient $C_{V}$ on the right-hand side of Eq. (49) is the heat capacity at fixed volume, while $\lambda$ is the latent heat, that is, the heat absorbed when the volume changes at fixed temperature.

Since $d Q=T d S$, it now follows from Eq. (2) that

$$
d U=C_{V} d T+(\lambda-P) d V
$$

and hence that

$$
\left(\frac{\partial C_{V}}{\partial V}\right)_{T}=\left(\frac{\partial(\lambda-P)}{\partial T}\right)_{V},
$$

or

$$
\left(\frac{\partial C_{V}}{\partial V}\right)_{T}=\left(\frac{\partial \lambda}{\partial T}\right)_{V}-\left(\frac{\partial P}{\partial T}\right)_{V} .
$$

On the other hand, since $d S=d Q / T$, we can write equally well rewrite Eq. 49, in the form

$$
d S=\frac{C_{V}}{T} d T+\frac{\lambda}{T} d V
$$

to obtain the expression

$$
\left(\frac{\partial\left(C_{V} / T\right)}{\partial V}\right)_{T}=\left(\frac{\partial(\lambda / T)}{\partial T}\right)_{V},
$$

from which it follows that

$$
\left(\frac{\partial C_{V}}{\partial V}\right)_{T}=\left(\frac{\partial \lambda}{\partial T}\right)_{V}-\frac{\lambda}{T}
$$

Comparison with Eq. 52 then shows that

$$
\lambda=T\left(\frac{\partial P}{\partial T}\right)_{V} .
$$

And substitution of the right-hand side for $\lambda$ in Eq. (55) yields an expression for the derivative of the heat capacity:

$$
\left(\frac{\partial C_{V}}{\partial V}\right)_{T}=T\left(\frac{\partial^{2} P}{\partial T^{2}}\right)_{V}
$$


Thermodynamics, as we can see, relates the heat capacity and latent heat to thermal derivatives of the pressure.

Our second illustration deals with the Enthalpy. We begin with an expression analogous to Eq. 49:

$$
d Q=C_{p} d T+\bar{\lambda} d P
$$

where $C_{p}$ is the heat capacity at constant pressure and $\bar{\lambda}$ is another latent heat, the heat absorbed as the pressure is varied at fixed temperature.

From Eqs. 10 and 49 we have that

$$
d H=C_{p} d T+(\bar{\lambda}+V) d P .
$$

Since the Enthalpy $H$ is an analytic function of its natural variable $T$ and $P$, we can see that

$$
\left(\frac{\partial C_{p}}{\partial P}\right)_{T}=\left(\frac{\partial(\bar{\lambda}+V)}{\partial T}\right)_{P},
$$

equivalent to the equality

$$
\left(\frac{\partial C_{p}}{\partial P}\right)_{T}=\left(\frac{\partial \bar{\lambda}}{\partial T}\right)_{P}+\left(\frac{\partial V}{\partial T}\right)_{P} .
$$

On the other hand, recalling that $d S=d Q / T$, we can rewrite Eq. 49 in the form

$$
d S=\frac{C_{p}}{T} d T+\frac{\bar{\lambda}}{T} d P,
$$

which implies that

$$
\left(\frac{\partial\left(\frac{C_{p}}{T}\right)}{\partial P}\right)_{T}=\left(\frac{\partial\left(\frac{\bar{\lambda}}{T}\right)}{\partial T}\right)_{P}
$$

a result that can be immediately reduced to the form

$$
\left(\frac{\partial C_{p}}{\partial P}\right)_{T}=\left(\frac{\partial \bar{\lambda}}{\partial T}\right)_{P}-\frac{\lambda}{T} .
$$

Comparison with Eq. 61 now shows that

$$
\bar{\lambda}=-T\left(\frac{\partial V}{\partial T}\right)_{P},
$$

and substitution of the right-hand side for $\bar{\lambda}$ in Eq. (64) yields the relation

$$
\left(\frac{\partial C_{p}}{\partial p}\right)_{T}=-T\left(\frac{\partial^{2} V}{\partial T^{2}}\right)_{P} .
$$

Similarly, the other thermodynamical potentials yield relations between physical properties of experimental interest.

\section{Concluding remarks}

Thermodynamics relates energy changes in physical systems to the changes in other physical properties. Capitally important is the First Law, which relates differential changes in the internal energy to the exact differential of the entropy and exact differentials of other variables. Examples are Eqs. (2) and (14). For definiteness, this summary will refer to the former, and hence consider $S$ and $V$ as the natural variables of $U$. We can then make the following statements.

3.1 Postulate. The Internal Energy $U=U(S, V)$, expressed as a function of its entropy $S$ and volume $V$, contains all thermodynamic information about a simple fluid system.

$U=U(S, V)$ can be inverted to yield $S=S(U, V)$, which likewise contains all the thermodynamic information about the system.

3.2 Changes of variables. Instead of the pair $(S, V)$, we may work with any of the pairs $(T, V),(S, P)$ or $(T, P)$. While the solution of equations such as (3) or (4) may be inverted to yield the entropy or volume as functions of other variables, and hence determine $U$ as functions of $P$ or $T$, information is lost in these algebraic transformations. Only with assistance of additional information can we recover the content of $U(S, V)$. The derivation of Eq. (37), aided by the Third Law of Thermodynamics, is an example.

3.3 Legendre transforms. The Internal Energy is a thermodynamic potential. The other thermodynamic potentials, defined as Legendre transforms of $U(S, V)$, are functions of other physical variables - their own natural variables - that preserve the information in the function $U(S, V)$. As illustrated by Eq. (23), the Legendre transform of a function is defined with respect to one of its variables. The Enthalpy $H(S, P)$ is the Legendre transform of $U(S, V)$ with respect to $V$, and the Helmholtz Free Energy $F(T, V)$ is the Legendre transform of $U(S, V)$ with respect to $S$, while the Gibbs Free Energy $G(T, P)$ can be obtained either as the Legendre transform of $F(T, V)$ with respect to $V$ or the Legendre transform of $H(S, P)$ with respect to $S$. Table 1 displays the definitions, natural variables, and partial derivatives of the four thermodynamic potentials.

As indicated in item 3.1, the function $U=U(S, V)$ can be inverted to yield $S=S(U, V)$. The Legendre transforms of $S(U, V)$ define the Massieu functions, which are equivalent to the thermodynamic potentials. As explained, for example, by Callen [2], Thermodynamics can be formulated either in the Energy representation $U(S, V)$ or in the equivalente Entropy representation $S=S(U, V)$. The Second Law of Thermodynamics requires the 
internal energy to be a convex function of its natural variables.

3.4 Void thermodynamic potential.

As explained in Sec. 2 simple considerations show that, if $U$ has $n$ natural variables, then the Legendre transform procedure yields $2^{n}$ distinct thermodynamic potentials. Table 1 ratifies this reasoning for $n=2$. For $n=3$, however, one of the eight potentials may be void, as we now discuss.

If we again consider a system with variable number of particles, Eq. (2) acquires the more general form

$$
d U=T d S-P d V+\mu d N
$$

and the Internal Energy becomes a function $U=$ $U(S, V, N)$. The last term on the right-hand side of Eq. 67) is the variation of Internal Energy when the number of particle changes by $d N$. On the basis of the Legendre Transformation procedure, it is then straightforward to define a thermodynamic potential that is a function of $T, P$ and $\mu$. Nonetheless, the resulting thermodynamic potential vanishes identically. For a discussion of this issue and its relation to the Euler Theorem, the reader is referred to Callen [2, page 148].

\section{References}

[1] D.P. Schroeder, An Introduction to Thermal Physics (Addison-Wesley, San Francisco, 2000).

[2] H.B. Callen, Thermodynamics and an Introduction to Thermostatistics (John Wiley \& Sons, New York, 1985), $2^{\mathrm{a}}$ ed.

[3] R. Baierlein, Thermal Physics (Cambridge University Press, Cambridge, 1999).

[4] J.P. Cannon, Am. J. Phys. 72, 753 (2004).

[5] R.B. Israel, Convexity in the Theory of Lattice Gases (Princeton University Press, Princeton, 1979).

[6] R.K.P. Zia, E.F. Redish and S.R. McKay, Am. J. Phys. 77, 614 (2009).

[7] C.J. Adkins, Equilibrium Thermodynamics (McGrawHill, London, 1968).

[8] V.V. Sychev, The Differential Equations of Thermodynamics (MIR, Moscow, 1983).

[9] Y.B. Rumer and M.S. Ryvkin, Thermodynamics, Statistical Physics and Kinetics (MIR, Moscow, 1980).

[10] W. Grimus, Ann. Physik 525, A32 (2013). 